\title{
A relevância do exame físico do idoso para a assistência de enfermagem hospitalar
}

\author{
The relevance of physical examination \\ of the elderly patient in the nursing assistance hospital
}

\author{
La relevancia del examen fisico del anciano \\ para la asistencia de enfermería hospitalar
}

Juliana Coutinho de Paula', Fernanda Aparecida Cintra²

\begin{abstract}
RESUMO
Introdução: O exame físico do idoso fornece dados relevantes para a assistência, contudo observa-se na prática de enfermagem que raramente ele é realizado de forma singular e sistematizado. Objetivo: foi verificar a importância atribuída por enfermeiros ao exame físico do idoso, para a assistência de enfermagem hospitalar. Método: foram entrevistados 25 enfermeiros das unidades de internação: Gastroclínica e Gastrocirurgia, Cardiologia e Pneumologia, e Enfermaria Geral de Adultos I e II do Hospital de Clínicas da Universidade Estadual de Campinas. Resultados: apenas um enfermeiro não foi contemplado com conteúdo sobre exame físico durante a graduação, e que para grande parte dos entrevistados os temas senescência e senilidade foram abordados de forma deficitária ou não foram abordados. A maioria dos entrevistados (88\%) realiza o exame físico e mostra-se motivada para efetuá-lo, embora o faça de maneira incompleta. Considerações finais: Os achados revelam a necessidade de capacitar os enfermeiros que lidam com idosos, tendo em vista as peculiaridades do exame físico e as necessidades dessa clientela.
\end{abstract}

Descritores: Exame físico; Idoso; Enfermagem geriátrica; Ensino

\begin{abstract}
Background: In nursing practice, it has been noticed that those examinations are rarely done in a singular and systematic way. Aim: To verify the importance attributed by nurses to the physical examination in elderly, for hospital nursing assistance. Method: Twenty five nurses from the following wards were interviewed: Surgery, Gastroenterology, Cardiology and Pneumology, and Adult General ward I - II at the Clinical Hospital, Campinas State University. Results: Only one of the informants had no information on physical examination during graduation and most of them had lack of information or no information at all on senescence and senility. Most informants (88\%) perform the physical examination and show motivation to execute it, though they do it in an incomplete way. Final Considerations: This results show the requirement of capacitation for nurses who treats the aged, considering that physical examination in elderly has its specificities.
\end{abstract}

Keywords: Physical examination; Age; Geriatric nursing; Teaching

\section{RESUMEN}

Introducción: El examen físico del anciano ofrece datos relevantes para la asistencia. Sin embargo, se observa que en la práctica de enfermería es poco realizado de forma sistemática. Objetivo: De este estudio fue verificar la importancia atribuida por los enfermeros al examen físico del anciano, para la asistencia de enfermería hospitalar. Método: Fueron entrevistados 25 enfermeros que integran el equipo de trabajo de los servicios de Gastroclínica y Cirugía, Cardiología y Neumología, y Enfermería General de Adultos I - II, del Hospital de Clínicas de la Universidad Estatal de Campinas. Resultados: Apenas un enfermero no recibió, en su curso de graduación, informaciones sobre el examen físico. Para la gran parte de los entrevistados el abordaje sobre envejecimiento y senilidad fue deficitario o no fue contemplado. La mayoría de los entrevistados (88\%) realiza el examen físico y se muestra motivada para efectuarlo, aunque lo hagan de manera incompleta. Conn Los datos obtenidos apuntan la necesidad de capacitación de los enfermeros que cuidan de los ancianos, considerando las particularidades de este examen y las necesidades de estos pacientes.

Descriptores: Examen físico; Anciano; Enfermería geriátrica; Enseñanza

1 Acadêmica de Enfermagem. Faculdade de Ciências Médicas. Universidade Estadual de Campinas - Unicamp - Campinas (SP),
Brasil. Trabalho de Iniciação Científica, financiado pelo Serviço de Apoio ao Estudante/Unicamp.
2 Professora Assistente da Faculdade de Ciências Médicas da Universidade Estadual de Campinas - Unicamp - Campinas (SP). 


\section{INTRODUÇÃO E JUSTIFICATIVA}

Com os avanços tecnológicos o homem conseguiu prolongar o seu tempo de vida e passou a vivenciar alterações estruturais e funcionais decorrentes do processo fisiológico de envelhecimento (senescência) ${ }^{(1-2)}$. Concomitante ao processo fisiológico de envelhecimento surgem outras alterações decorrentes de doenças crônicas, comumente manifestadas nas pessoas com idade superior a 60 anos (senilidade) ${ }^{(1-4)}$. No decorrer dos processos de senescência e senilidade, o grau de dependência dos idosos tende a aumentar gradativamente, exigindo uma assistência de enfermagem apropriada e eficaz, crescente em quantidade e qualidade, capacitada para atender às necessidades individuais e coletivas, e às particularidades da idade ${ }^{(3,5-6)}$.

A assistência de enfermagem baseia-se em uma estrutura lógica de ações denominada Sistematização da Assistência de Enfermagem (SAE), a qual é composta pelas seguintes etapas: histórico, obtido pela entrevista e pelo exame físico; diagnóstico de enfermagem; prescrição de enfermagem; e, evolução de enfermagem ${ }^{(7-8)}$.

$\mathrm{O}$ exame físico representa um instrumento de grande valia para a assistência, uma vez que permite ao enfermeiro realizar o diagnóstico e planejar as ações de enfermagem, acompanhar e avaliar a evolução do paciente ${ }^{(3,9-10)}$.

Nos idosos o exame físico deve ir além da avaliação clínica. Esses pacientes são mais susceptíveis a desenvolver limitações para as atividades de vida diárias e manifestar as chamadas "Síndromes Geriátricas", como "imobilidade, incontinência, uso incorreto de medicação, alterações cognitivas, perda de peso e depressão" (11). Assim, os mínimos sinais e sintomas devem ser observados e interpretados, fundamentando o diagnóstico de enfermagem e a elaboração de um plano de cuidados específico e individualizado, a fim de possibilitar uma assistência que garanta a manutenção e a promoção da saúde do idoso, bem como a sua independência ${ }^{(3,5)}$.

Em nossa vivência acadêmica observamos que os enfermeiros não realizam regularmente e de forma sistematizada o exame físico dos pacientes adultos e, particularmente, dos idosos. Observação semelhante foi evidenciada em outro estudo, no qual apenas um dos 34 idosos entrevistados mencionou ter sido submetido ao exame físico realizado pelo enfermeiro; os demais não souberam informar ou não se recordaram ${ }^{(6)}$. A esse respeito, outros autores chamam a atenção para a falta de instrumentalização da equipe de enfermagem para o cuidado do idoso, que possui características específicas do processo de envelhecimento ${ }^{(3,12)}$.

A falta ou a realização inadequada do exame físico no idoso hospitalizado, ao nosso ver, dificulta a assistên- cia de enfermagem individualizada e voltada para as necessidades desse sujeito, uma vez que muitas das alterações decorrentes do processo de envelhecimento deixam de ser identificadas. Esta lacuna na identificação de tais alterações, por sua vez, dificulta o planejamento e a implementação de ações que contribuem para a redução do tempo de internação e do número de reinternações hospitalares.

Esta problemática levou-nos a realizar o presente estudo, com o intuito de responder aos seguintes questionamentos:

1. Os enfermeiros realizam o exame físico dos idosos hospitalizados? Qual a contribuição deste exame para a assistência de enfermagem?

2. Os enfermeiros sentem-se motivados para realizar o exame físico dos idosos hospitalizados?

Acreditamos que a identificação dos fatores relacionados à falta ou à realização inadequada do exame físico em idosos hospitalizados fornecerá subsídios para o desenvolvimento e o aprimoramento da assistência de enfermagem voltada às especificidades desta faixa etária. Esperamos, ainda, que este estudo amplie as reflexões sobre a Enfermagem Geriátrica e Gerontológica, na perspectiva de sua maior projeção e fundamentação científica.

\section{OBJETIVOS}

\section{Geral}

Verificar a relevância atribuída pelos enfermeiros ao exame físico do idoso, para a assistência de enfermagem hospitalar.

\section{Específicos}

1. Caracterizar o exame físico do idoso hospitalizado, segundo os enfermeiros que realizam a assistência de enfermagem;

2. Identificar os dados do exame físico que os enfermeiros coletam e utilizam na assistência de enfermagem ao idoso hospitalizado;

3. Verificar os significados que os enfermeiros atribuem ao exame físico para a assistência ao idoso hospitalizado;

4. Verificar se os enfermeiros sentem-se motivados para realizar o exame físico no idoso hospitalizado.

\section{MATERIALE MÉTODO}

O estudo foi realizado no Hospital de Clínicas da Universidade Estadual de Campinas (Unicamp), Campinas, São Paulo, nas unidades de internação: Gastroclínica e Gastrocirurgia; Cardiologia e Pneumologia; e Enfermaria Geral de Adultos I e II, as quais concentravam o maior número de idosos ${ }^{(6)}$. 
Fizeram parte do estudo 25 dos 41 enfermeiros lotados nas unidades de internação citadas, que atenderam aos seguintes critérios: 1- exercício das atividades profissionais como enfermeiro nesses locais, em período superior a seis meses; e, 2- concordância em participar do estudo e obtenção da assinatura do Termo de Consentimento Livre e Esclarecido.

Os dados foram coletados por meio de entrevistas individuais, realizadas pela pesquisadora em horários préagendados. Foi utilizado um instrumento próprio (Anexo I) contendo questões abertas e fechadas, e composto pelas seguintes partes: I - Identificação dos enfermeiros; II - Informações relacionadas ao conhecimento teóricoprático dos enfermeiros; e, III - Informações sobre o exame físico dos idosos e a assistência de enfermagem.

A coleta de dados foi realizada no período de dezembro de 2002 a março de 2003, após aprovação pelo Comitê de Ética em Pesquisa da Faculdade de Ciências Médicas da Unicamp, sob o parecer $n^{\circ} 460 / 2002$.

Previamente à entrevista, os enfermeiros foram orientados a considerar como idosos os pacientes com idade igual ou superior a 60 anos, conforme estabelece a Organização Mundial de Saúde ${ }^{(13)}$.

Os dados obtidos foram organizados, distribuídos e analisados em frequiência absoluta e relativa.

\section{RESULTADOS E DISCUSSÃO}

Dos 41 enfermeiros lotados nas unidades de internação eleitas para este estudo, apenas $25(60,97 \%)$ foram entrevistados. Cinco (12,19\%) apresentaram inviabilidade para agendar a entrevista devido à dupla jornada de trabalho e à sobrecarga de atividades; quatro $(9,75 \%)$ ocupavam cargos administrativos; quatro $(9,75 \%)$ atuavam nas respectivas unidades há menos de seis meses; e três enfermeiros $(7,31 \%)$ recusaram-se a participar do estudo.

Dos 25 entrevistados, 16 (64\%) estavam na faixa etária entre 41 e 50 anos; 21 (84\%) eram do sexo feminino; e 11 (44\%) enfermeiros, o que correspondia à maioria, exerciam as atividades profissionais nas unidades de internação no período entre um e cinco anos.

Com respeito à formação acadêmica, 19 (76\%) enfermeiros graduaram-se em instituições privadas e seis (24\%) em instituições públicas. O tempo de titulação variou entre um e 23 anos, com a maior concentração dos profissionais (28\%) titulados entre 16 e 20 anos. Somente seis (24\%) dos 25 enfermeiros concluíram cursos de pós-graduação (stricto sensu ou latu sensu).

A abordagem de conteúdo sobre exame físico durante a graduação foi apontada por $24(96 \%)$ enfermeiros; destes, $19(79,16 \%)$ afirmaram que o conteúdo foi insuficiente para as atividades práticas. Esta insuficiência também foi identificada em estudo sobre o emprego de bases propedêuticas para o exame físico geral de enfermagem $^{(14)}$. Em contra-partida, em investigação realizada com enfermeiras de Unidade de Terapia Intensiva as autoras obtiveram que para a maioria $(32,3 \%)$ a prática profissional constituía o momento de maior significado para a aprendizagem do exame físico ${ }^{(15)}$. Embora reconheçamos a relevância da prática profissional, concordamos com a necessidade de uma fundamentação teórico-prática relacionada à propedêutica na formação acadêmica ou, pelo menos, nos cursos de pós-graduação ${ }^{(14)}$.

As alterações relacionadas a senescência durante a graduação foram contempladas para $13(52 \%)$ dos 25 enfermeiros entrevistados; destes, oito $(61,53 \%)$ afirmaram que o conteúdo foi insuficiente para a assistência de enfermagem. Em relação à senilidade, 19 (76\%) dos 25 enfermeiros afirmaram que o tema foi contemplado durante o curso de graduação, dos quais 14 $(73,68 \%)$ relataram que ele foi insuficiente para a prática profissional.

O aumento da população idosa exige que os profissionais da área da saúde, inclusive os enfermeiros, conheçam o processo de envelhecimento natural e as afecções associadas, de forma a estarem aptos para prestar uma assistência voltada às peculiaridades dessa população ${ }^{(2,16-17)}$. Considerando, em nosso estudo, que somente uma parcela dos entrevistados foi contemplada com os temas senescência e a senilidade na graduação, e que a sua maioria reconheceu-os insuficientes para a prática profissional, acreditamos que uma base sólida de conteúdos relacionados a esses temas na formação acadêmica contribuiria para formar profissionais melhor capacitados para o cuidado dos idosos.

Vinte e quatro (96\%) enfermeiros afirmaram realizar o exame físico nos idosos hospitalizados. Destes, 16 $(66,66 \%)$ disseram que realizam esse exame na admissão e diariamente durante a internação; quatro $(16,66 \%)$ o fazem durante a internação, sem citar a admissão; dois $(8,33 \%)$ realizam o exame somente na admissão; um $(4,16 \%)$ na admissão e na manifestação de alterações pelo paciente; e apenas um enfermeiro $(4,16 \%)$ afirmou que o faz na admissão, diariamente e na alta hospitalar.

Tendo como pressuposto que o exame físico fundamenta a assistência sistematizada de enfermagem, e que ela compreende o período desde a admissão até a alta do paciente, chama a atenção em nosso estudo que somente um enfermeiro afirmou realizar esse procedimento desde a admissão até a alta hospitalar.

Dos 24 (96\%) enfermeiros que afirmaram realizar o exame físico, $23(95,83 \%)$ revelaram que examinam os idosos pelo menos uma vez durante o plantão; destes, $10(43,47 \%)$ o fazem com maior frequiência, se necessário. Dentre as justificativas para a prática do exame físico, as mais citadas foram: para "elaborar um plano 
de cuidados/prescrição de enfermagem" e para "realizar a evolução de enfermagem". Nenhum profissional relacionou esta prática às especificidades dos idosos, o que pode ser decorrente da falta de conteúdo sobre senescência e senilidade, ou da sua insuficiência na formação acadêmica dos entrevistados.

$\mathrm{Na}$ análise dos dados que os enfermeiros afirmaram coletar no exame físico dos idosos, os itens mais citados foram: peso, altura, nível de consciência, deambulação/marcha, padrão respiratório, ausculta pulmonar e cardíaca, frequiência cardíaca e freqüência das eliminações. Concomitantemente, verificamos uma grande diversidade nos itens coletados, semelhante à encontrada em outro estudo e atribuída pelas autoras à "dificuldade de estabelecer o que deve ou não ser incluído no exame físico"(15). Isso mostra a falta de uma estrutura sólida de conhecimentos para a realização do exame físico de enfermagem que aponte quais itens devem ser privilegiados pelo enfermeiro, a fim de obter subsídios para a sistematização da assistência de enfermagem e o direcionamento desta assistência ${ }^{(18)}$.

Sobre a utilização das informações coletadas por meio do exame físico, $15(62,5 \%)$ dos 24 enfermeiros afirmaram utilizar todos os dados obtidos; os demais apontaram o uso de somente parte desses dados. Embora as informações obtidas pelo exame físico fossem parcialmente utilizadas, $14(58,33 \%)$ dos 24 enfermeiros que realizavam essa prática reconheceram que essas informações eram suficientes para a assistência de enfermagem aos idosos. O reconhecimento da suficiência de informações pode estar associado à necessidade de uma melhor instrumentalização destes profissionais para a obtenção dos dados, interpretação e utilização na assistência de enfermagem. Na literatura encontramos que o conhecimento dos enfermeiros sobre as bases propedêuticas para o exame físico revela-se insuficiente para a necessidade manifestada pelos mesmos, o que reitera a nossa compreensão sobre a relevância da capacitação dos profissionais ${ }^{(14)}$.

Ao questionarmos os enfermeiros sobre o que representa o exame físico para a assistência de enfermagem, os significados que mais se destacaram foram: prática que permite a individualização do paciente, com ênfase para as necessidades e peculiaridades; "é o fio condutor" de toda a assistência, uma vez que revela o que não é expresso verbalmente pelo idoso; recurso para "melhorar a assistência e tornar a hospitalização mais amena"; e, instrumento para "iniciar o cuidado com respeito, dignidade e carinho".

No contexto hospitalar observamos, com freqüência, que os idosos mantêm-se isolados, tristes, pouco comunicativos e sem verbalizar suas queixas, inquietações e medos. Nesse sentido, o exame físico representa uma oportunidade para o enfermeiro amenizar o des- conforto e gerar confiança nos pacientes e possibilita, assim, ao idoso expressar os seus sentimentos e as suas queixas, e "tornar a hospitalização mais amena" (19).

Dentre os 25 enfermeiros entrevistados, 22 (88\%) mostraram-se motivados para realizar o exame físico dos idosos. As principais justificativas foram: "é importante para a assistência de enfermagem" e "é necessário para a assistência de enfermagem". Dos três (12\%) enfermeiros que revelaram falta de motivação, um relacionou a desmotivação à sobrecarga de atividades, outro à obrigatoriedade do procedimento, e outro à necessidade de registro dos dados. A sobrecarga de atividades parece contribuir não apenas para desmotivar a prática do exame físico como também de toda a SAE, a ponto de levar os profissionais a não executá-la. Um estudo sobre os métodos avaliativos da assistência de enfermagem em instituições hospitalares mostrou que, segundo as enfermeiras responsáveis por diferentes serviços, "a carência de pessoal, o desinteresse das enfermeiras e a falta de tempo referida pelas mesmas" foram os principais motivos para a não implantação da SAE em 10 dos 18 hospitais selecionados na pesquisa ${ }^{(20)}$.

Os significados atribuídos pelos enfermeiros ao exame físico e a motivação expressada para realizá-lo revelam a importância desta prática para o exercício profissional. Contudo, a diversidade dos itens avaliados, o reconhecimento da utilização de apenas parte desses itens para a assistência de enfermagem, e a divergência quanto aos momentos da realização do exame físico, mostram a necessidade de capacitação desses profissionais para uma avaliação clínica cuidadosa, para a correta identificação dos problemas dos idosos e para a utilização destes problemas na SAE.

A esse respeito, recomenda-se que as chefias de enfermagem dos hospitais procurem facilitar aos enfermeiros a sua participação em cursos de pós-graduação, que contemplem as bases propedêuticas ${ }^{(14)}$. Acrescentamos a isso, a instrumentalização desses profissionais para o cuidado do idoso, tendo em vista a carência de conteúdo sobre o processo de envelhecimento e as alterações decorrentes desse processo nos cursos de graduação.

Acreditamos que estes e outros cursos, como os de extensão, ou ainda os de treinamento em serviço, poderão instrumentalizar os enfermeiros para a SAE, particularmente para a assistência ao idoso hospitalizado.

\section{CONSIDERAÇÕES FINAIS}

O cuidado ao idoso durante a hospitalização merece atenção de todos os profissionais que participam deste cuidado. $\mathrm{O}$ exame físico representa uma das etapas que sustenta a Sistematização da Assistência de Enfermagem. Para tanto, é preciso o conhecimento de todos os pas- 
sos necessários para a sua efetivação. Sem isso, continuaremos realizando essa prática sem fundamentação científica e sem a relevância que ela remete.

\section{REFERÊNCIAS}

1. Vieira CM, Glashan, RQ. Aspectos gerais da anatomia e fisiologia do envelhecimento - uma abordagem para o enfermeiro. Acta Paul Enferm. 1996;9(3):24-30.

2. Jacob Filho W. Envelhecimento e atendimento domiciliário. In: Duarte YAO, Diogo MJD'E, organizadores. Atendimento domiciliar: um enfoque gerontológico. São Paulo: Atheneu; 2000. p.19-26.

3. Marin MJS. Preparando o idoso para a alta hospitalar [tese]. Ribeirão Preto (SP): Escola de Enfermagem de Ribeirão Preto, Universidade de São Paulo; 1999.

4. Andrade OG. Suporte ao sistema de cuidado familiar ao idoso com acidente vascular cerebral a partir de uma perspectiva holística de saúde [tese]. Ribeirão Preto (SP): Escola de Enfermagem de Ribeirão Preto,Universidade de São Paulo; 2001.

5. Horta WA. A assistência de enfermagem ao adulto idoso. Enf Novas Dimens. 1978;4(5): 268-73.

6. Ribeiro GLMT. O processo de admissão hospitalar do idoso em unidade de clínica médica [tese]. Campinas (SP): Faculdade de Educação da Universidade Estadual de Campinas; 2001.

7. Conselho Regional de Enfermagem de São Paulo(COREN-SP) . Decisão COREN-SP/DIR/008/99. Normatiza a implementação da sistematização da Assistência de Enfermagem-SAE, nas Instituições de saúde, no âmbito do Estado de São Paulo. CORENSP. 2000;(26):12-3.

8. Du Gas WB. Práticas de enfermagem. 2a ed. Rio de Janeiro: Guanabara Koogan; 1986.

9. Campedelli MC. Consulta de Enfermagem em geriatria: tópicos a serem abordados no exame físico: parte II. Rev Esc Enferm USP. 1992;26(1):33-42.

10. Gaidzinski RR, Kimura M. Entrevista e exame físico - instrumentos para o levantamento de dados. In: Campedelli MC, organizadora. Processo de enfermagem na prática. São Paulo: Ática; 1989. p. 66-88.
11. Diogo MJD'E, Paschoal SMP, Cintra FA. Avaliação global do idoso. In: Duarte YAO, Diogo MJD’E, organizadores. Atendimento domiciliar: um enfoque gerontológico. São Paulo: Atheneu; 2000. p.145-71.

12. Cintra FA, Sawaia BB. A significação do glaucoma e a mediação dos significados de velhice na perspectiva vygotskiana: subsídios para a educação à saúde. Rev Esc Enferm USP. 2000; 34(4):339-46.

13. Organización Mundial de la Salud(OMS). Planificación y organización de los servicios geriátricos: informe de un comité de expertos. Genebra; 1974. (Série de Informes Técnicos, 548).

14. Barros ALBL, Glashan RQ, Michel JLM. Bases propedêuticas para a assistência de enfermagem: uma necessidade atual. Acta Paul Enferm. 1996; 9(1):28-37.

15. Kimura M, Miyadahira AMK, Cruz DALM, Takahashi EIU, Padilha KG, Sousa RMC. O exame físico e o enfermeiro de UTI. Rev Esc Enferm USP. 1994;28(2): 156-70.

16. Diogo MJD'E. Consulta de enfermagem em gerontologia. In: Papaléo Netto M. Gerontologia. São Paulo: Atheneu; 1996. p. 209-21.

17. Duarte YAO. Princípios da assistência de enfermagem gerontológica. In: Papaléo Netto M. Gerontologia. São Paulo: Atheneu; 1996. p. 222-29.

18. Laganá MTC, Friedlander MR, Pimenta CAM, Silva MJP, Imanich RM. Características específicas do exame físico realizado pelo enfermeiro. Rev Esc Enferm USP. 1993;27(1):95-9.

19. Mattos MD, Santos SRO. Exame físico: uma proposta de ensino para o curso de especialização em centro de terapia intensiva. Nursing. 1997;1(7):12-8. [edição brasileira]

20. Montes ADAS, Adami NP, Barros ALBL. Métodos avaliativos da assistência de enfermagem em instituições hospitalares. Acta Paul Enferm. 2001;14(1):89-97. 


\section{ANEXO I \\ INSTRUMENTOPARA COLETADEDADOS}

Data:

\section{PARTEI-IDENTIFICAÇÃO}

Iniciais:___ Idade: _ _ anos Sexo: ( ) Feminino ( ) Masculino

Unidade de Internação:

- Curso de Graduação em Enfermagem:

Instituição:

Ano de conclusão:

- Curso de Pós-graduação: ( ) Não （ ) Sim

Se Sim:

Instituição:

Maior titulação:

Ano de conclusão:

Área:

\section{PARTEII-INFORMAÇÕESRELACIONADASAOCONHECIMENTOTEÓRICO-PRÁTICONAFORMAÇÃOPROFISSIONAL}

1- No curso de graduação, foi abordado algum conteúdo sobre exame físico? ( ) Não （ ) Sim

Se $\underline{\operatorname{Sim}}$, o conteúdo abordado foi suficiente para a sua prática profissional atual? ～～） Não （ ) Sim

2- No curso de graduação, foi abordado algum conteúdo sobre as alterações:

a. relacionadas à senescência (processo fisiológico do envelhecimento): ( ) Não （ ) Sim

Se $\underline{\operatorname{Sim}}$, o conteúdo foi suficiente para a sua prática profissional atual? ( ) Não （ ) Sim

b. relacionadas à senilidade (envelhecimento associado às afecções): ( ) Não （ ）Sim

Se $\underline{\operatorname{Sim}}$, o conteúdo foi suficiente para a sua prática profissional? ( ) Não （ ）Sim

\section{PARTE III - INFORMAÇÕESSOBRE OEXAME FÍSICO DO IDOSO EAASSISTÊNCIADE ENFERMAGEM}

1- Você realiza o exame físico dos idosos internados? ( ) Não （ ） Sim Justifique:

Se $\underline{\operatorname{Sim}}$ :

1.1- Em que fase (s) da internação você realiza esse exame físico?

1.2- Com que freqüência, no plantão, você realiza o exame físico do idoso?

1.3-No exame físico do idoso, identifique que dados você coleta nos seguintes segmentos e sistemas:

- Medidas Antropométricas:

- Pele e Fâneros:

- Sistema Neurológico e Estado Mental:

- Sistema Músculo - Esquelético:

- Sistema Linfático:

- Sistema Respiratório:

- Sistema Cardiovascular:

- Sistema Gênitourinário:

- Trato Gastrointestinal:

1.4-Dos dados coletados no exame físico do idoso, que informações você utiliza para a assistência de enfermagem?

1.5- Você considera que as informações que utiliza do exame físico do idoso são suficientes para a assistência de enfermagem?

( ) Não ( ) Sim Justifique:

1- O que representa o exame físico do idoso para a assistência de enfermagem?

2- Você sente-se motivado para realizar o exame físico do idoso hospitalizado? ( ) Não （ ) Sim

Justifique:

OBSERVAÇÕES DAENTREVISTADORA: 